\title{
Percutaneous coronary intervention for the treatment of unprotected left main coronary artery disease
}

\author{
Laxman Dubey MD DM, Rabindra Bhattacharya MD DM, Gangapatnam Subramanyam MD DM
}

L Dubey, R Bhattacharya, G Subramanyam. Percutaneous coronary intervention for the treatment of unprotected left main coronary artery disease. Curr Res: Cardiol 2014;1(2):89-92.

Left main coronary artery disease is associated with poor clinical outcomes. Coronary artery bypass graft surgery has been the standard treatment for unprotected left main coronary artery disease. However, with the introduction of drug-eluting stents and advances in catheter techniques, together with advances in periprocedural adjunctive pharmacotherapies,

$\mathrm{T}$ he prevalence of left main coronary artery (LMCA) disease is low.

Approximately $4 \%$ to $7 \%$ of patients with acute myocardial infarction (MI) undergoing coronary angiography (CAG) have significant involvement of the LMCA $(1,2)$. Patients with LMCA disease are at high risk for cardiovascular events because occlusion of this vessel compromises flow to at least $75 \%$ of the left ventricle and 100\% in cases of the left dominant type. As a result, severe LMCA disease reduces flow to a considerable portion of the myocardium, placing the patient at high risk for life-threatening events such as left ventricular dysfunction and arrhythmias $(3,4)$. Patients with unprotected LMCA disease treated medically have a three-year mortality rate of $50 \%$ (3). Although coronary artery bypass graft (CABG) surgery has been considered the 'gold standard' for unprotected LMCA revascularization, percutaneous coronary intervention (PCI) has recently emerged as a possible alternative mode of revascularization in carefully selected patients. The current American College of Cardiology Foundation (ACCF)/American Heart Association (AHA)/Society for Cardiovascular Angiography and Interventions (SCAI) guidelines also state that PCI for LMCA disease is a reasonable alternative to CABG in patients who have anatomical conditions associated with good procedural and longer-term outcomes, and who are at increased risk for surgery (5).

\section{CASE PRESENTATION}

A 76-year-old man with a history of systemic hypertension presented to the emergency department of the authors' institution with acute-onset left-sided chest pain and shortness of breath of $1 \mathrm{~h}$ duration. The patient was a past smoker. In the emergency department, his blood pressure (BP) was $120 / 80 \mathrm{mmHg}$, with a heart rate of 85 beats $/ \mathrm{min}$. The initial electrocardiogram showed sinus rhythm at a heart rate of 90 beats/min with ST depression in leads II, III, aVF, V4, V5 and V6. He was diagnosed with acute coronary syndrome (ACS), admitted to the coronary care unit and managed with low-molecular-weight heparin, acetylsalicylic acid, clopidogrel, beta-blockers and atorvastatin.

Thirty minutes later, the patient complained of increased intensity in chest pain and difficulty breathing. His systolic BP decreased to $60 \mathrm{mmHg}$ with an undetectable diastolic BP. A dopamine drip at a rate of $15 \mu \mathrm{g} / \mathrm{kg} / \mathrm{min}$ was started and the patient was transferred to the catheterization laboratory for CAG. CAG was performed via right femoral artery approach, which revealed approximately $90 \%$ stenosis in the mid part of the LMCA (Figures 1A and 1B), 70\% stenosis in the mid segment of the left anterior descending (LAD) artery and $70 \%$ to percutaneous coronary intervention has become a viable alternative to coronary artery bypass graft for the management of this complex coronary lesion. The authors describe percutaneous coronary intervention in the treatment of unprotected left main coronary artery disease and briefly review its role in the management of patients with the condition.

Key Words: Left main coronary artery disease; Percutaneous coronary intervention; Revascularization

$80 \%$ stenosis in the proximal right coronary artery. The left circumflex coronary (LCX) artery was normal.

During the procedure, his BP remained low; a drip of noradrenaline was also started. Relatives of the patient were counselled about the disease, its prognosis and the need for emergency CABG. Because CABG surgery was not available at the authors' centre and the patient was in cardiogenic shock, a decision was made to perform PCI of the LMCA. Relatives of the patient agreed to PCI, and written and informed consent were obtained.

The LMCA was cannulated with a $3.5 \mathrm{~cm} 7 \mathrm{Fr}$ left Judkins guide catheter. The lesion was crossed with two balanced middle weight guidewires, one each in the LAD and LCX coronary arteries. Subsequently, a drug-eluting stent (DES), measuring $3.5 \mathrm{~mm} \times 16 \mathrm{~mm}$, was deployed in the LMCA, which was subsequently postdilated with a noncompliant balloon measuring $4.0 \mathrm{~mm} \times 10 \mathrm{~mm}$ (Figures $1 \mathrm{C}$ and

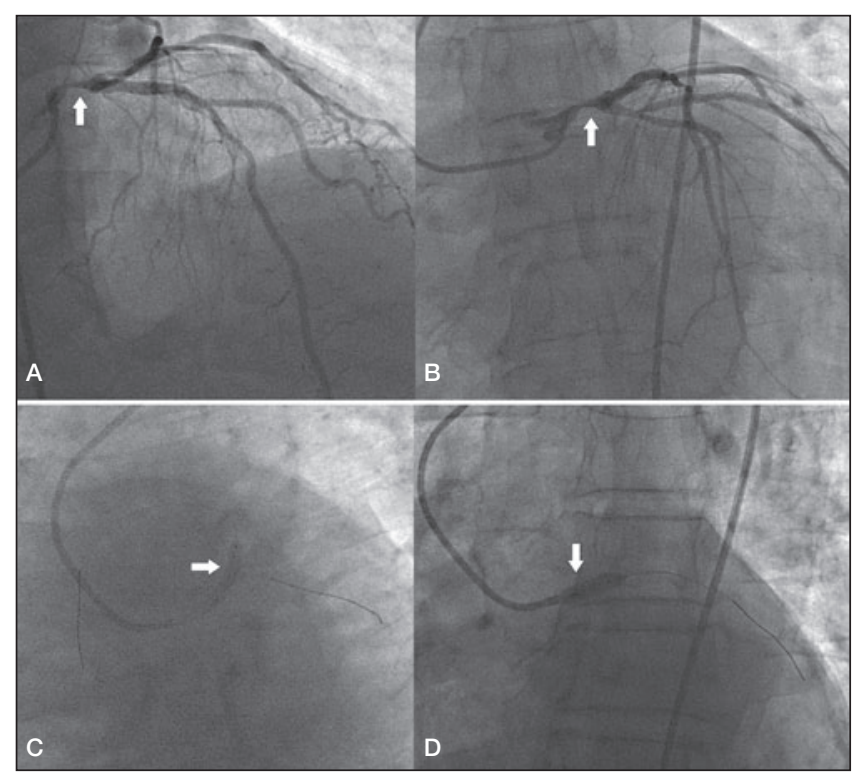

Figure 1) A and B Coronary angiography showing significant stenosis of the mid part of the left main coronary artery. C Stent deployment in the left main coronary artery. D Postdilation with a noncompliant balloon

Department of Cardiology, College of Medical Sciences and Teaching Hospital, Bharatpur-10, Chitwan, Nepal

Correspondence: Dr Laxman Dubey, Department of Cardiology, College of Medical Sciences and Teaching Hospital, Bharatpur-10, Chitwan, Nepal.

Telephone 977-9851123288, e-mail dubeylax@yahoo.com 


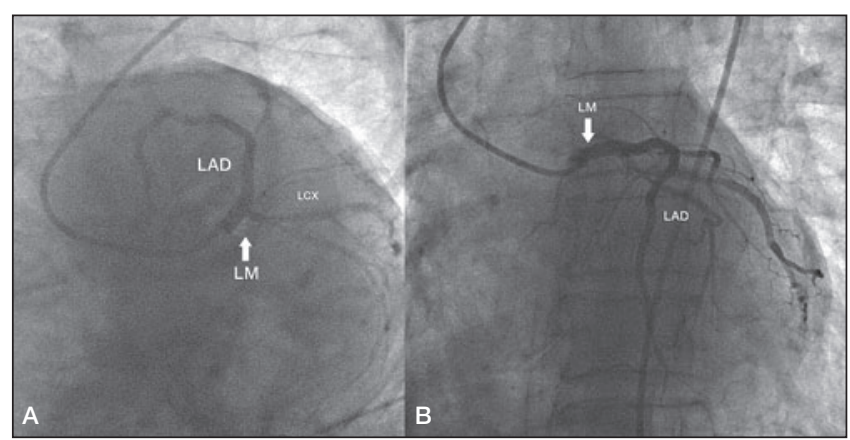

Figure 2) A and B Final result after stent deployment in the left main (LM) coronary artery and the left anterior descending (LAD) artery. LCX Left circumflex coronary artery

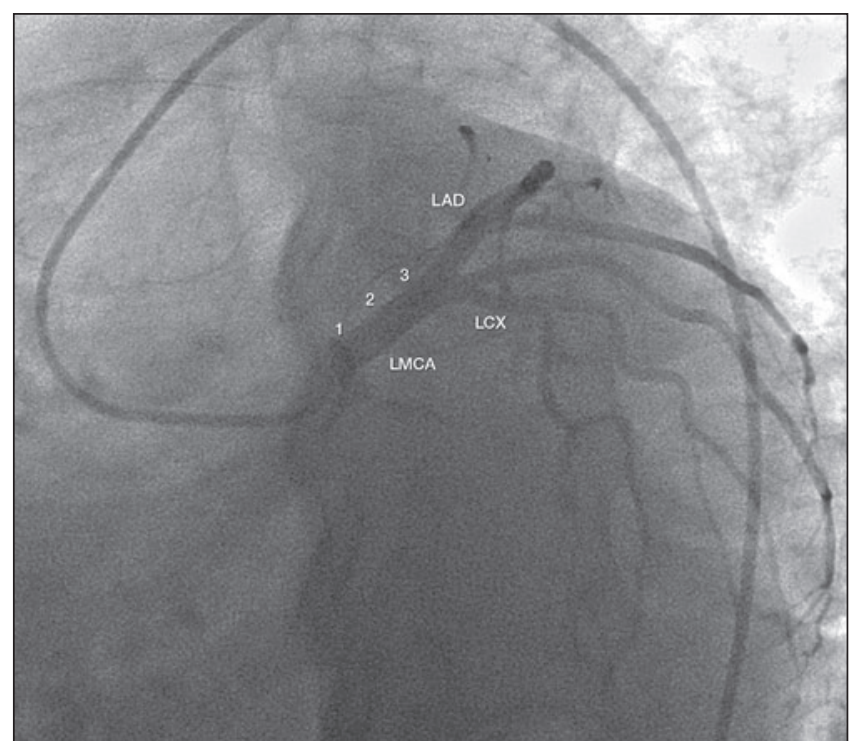

Figure 3) Three regions of the left main coronary artery (LMCA): 1 ostium; 2 Midshaft; 3 Distal portion. LAD Left anterior descending artery; LCX Left circumflex coronary artery

1D). Another DES, measuring $3.0 \mathrm{~mm} \times 33 \mathrm{~mm}$, was then deployed in the mid LAD artery. Final results showed good distal flow with thrombolysis in myocardial infarction III flow (Figures 2A and 2B). The procedure was uneventful and the patient's BP increased to $110 / 60 \mathrm{mmHg}$. The noradrenaline drip was discontinued and the dopamine dose was tapered.

The patient was transferred to the coronary care unit, where he was further managed with low-molecular-weight heparin, acetylsalicylic acid, clopidogrel, beta-blockers, angiotensin-converting enzyme inhibitor and atorvastatin. The patient's subsequent stay in hospital was uneventful and he was discharged five days later free of angina. At the two-year follow-up, the patient remained symptom free.

\section{ANATOMY OF THE LMCA}

The LMCA arises from the left sinus of Valsalva and is the first branch of the aorta. The diameter of the LMCA is usually $3.5 \mathrm{~mm}$ to $4.5 \mathrm{~mm}$, with a length of $10 \mathrm{~mm}$ to $20 \mathrm{~mm}$, but can extend to a length of $40 \mathrm{~mm}$. Because the LMCA supplies $75 \%$ of the left ventricle in patients with right dominant type and $100 \%$ in the case of left dominant type, severe LMCA disease would reduce flow to a considerable portion of the myocardium, placing the patient at high risk for life-threatening left ventricular dysfunction, arrhythmias and sudden death $(3,4)$.

The LMCA has three regions: the ostium, the midshaft and the distal portion (Figure 3). The distal portion of the LMCA bifurcates into the LAD and LCX arteries and, sometimes, trifurcates, resulting in the ramus intermedius (in approximately $22 \%$ of the population) as an additional branch. It is estimated that $1 \%$ of the population lacks an LMCA, and both arteries (LAD and LCX) arise from separate origins of the aorta. A reduction of at least $50 \%$ in the luminal diameter of the LMCA is considered to be significant LMCA disease. Unprotected LMCA disease is defined as significant stenosis in the LMCA and no previous CABG or patent bypass grafts to the LAD or LCX arteries.

\section{REVASCULARIZATION OF LMCA STENOSIS}

According to current ACCF/AHA/SCAI guidelines, unprotected LMCA disease is an absolute indication for surgical revascularization (ie, CABG), regardless of other arteriosclerotic lesions, both to alleviate symptoms and prognosis improvement (class I) (5). However, the current guidelines also state that PCI for LMCA disease is a reasonable alternative to $\mathrm{CABG}$ in patients who have anatomical conditions associated with good procedural and longer-term outcomes, and who are at increased risk for surgery (5). In the era of the balloon angioplasty, due to disappointing results and a higher rate of early death, LMCA stenosis was considered to be a contraindication (class III) to angioplasty. The evolution of DES has been a major breakthrough in PCI of LMCA stenosis, which has led to significant reduction in restenosis and target lesion revascularization compared with initial experiences with baremetal stents (BMS), which are limited by higher rates of restenosis and, in some series, sudden deaths (6). Three single-centre studies (7-9) showed high procedural success rates, low procedural complication rates and encouraging long-term outcomes for PCI for LMCA disease. The results were confirmed by the French multicentre Registry for stenting uNprotecteD LMCA stenosis (FRIEND) study (10).

\section{DES versus BMS}

Angiographic and procedural success do not differ significantly between DES and BMS in cases of PCI for LMCA disease. Given the lower rates of restenosis reported with DES in PCI of standard coronary lesions, there has been a trend toward their use in PCI for unprotected LMCA disease. Dores et al (11) reported that in high-risk populations, PCI for unprotected LMCA disease was safe and effective, with acceptable longterm clinical results and, thus, this strategy may be considered to be a valid alternative to CABG. They reported a high rate of major adverse cardiovascular events (MACE) in the first year, especially in BMStreated patients, suggesting the need for routine control angiography in all patients in whom a BMS is implanted and, whenever possible, that a DES should be used as the first choice (11).

A meta-analysis of observational studies and randomized controlled trials demonstrated lower crude event rates for DES than BMS for mortality, repeat revascularization, and MACE at six to 12 months, two years and three years (12). The reason for the lower mortality rate in the DES group in that meta-analysis was not clear. It may be that the lower rates of restenosis in DES provides a true advantage over BMS (12). These data support a strategy of DES for LMCA PCI except in cases with large reference vessel diameter or in which long-term dual antiplatelet therapy is contraindicated. DES implantation may be risky due to acute stent thrombosis, which can result in sudden cardiac death in $40 \%$ of patients (13-15).

\section{PCI versus CABG surgery}

To date, a large body of data from observational registries of clinical trials has compared the efficacy of PCI with CABG for the treatment of LMCA disease and supports the feasibility, efficacy and safety of stenting compared with CABG for the treatment of unprotected LMCA disease. The Study of Unprotected Left Main Stenting Versus Bypass Surgery (LE MANS) (16) enrolled 105 patients with $>50 \%$ LMCA narrowing, with or without multivessel coronary artery disease, who were equally suitable for PCI or CABG. That study showed that the risk for major adverse cardiac and cardiovascular events (MACCE) at 30 days was lower with PCI, whereas the risk of MACCE at one year was similar in PCI and CABG groups. At a longer-term mean $( \pm \mathrm{SD})$ 
follow-up of 28.0 \pm 9.9 months, there was a trend toward better survival with PCI. The Synergy Between Percutaneous Coronary Intervention with TAXUS and Cardiac Surgery (SYNTAX) trial (17) provided the largest randomized data from which to assess the early and longer-term safety and efficacy of PCI for LMCA disease. In this trial, PCI demonstrated a one-year rate of MACCE, death, MI or stroke similar to those observed after CABG; however, a higher rate of target vessel revascularization was found in the DES arm.

Results from the Revascularisation for Unprotected Left main Coronary Artery Stenosis: Comparison of Percutaneous Coronary Angioplasty versus Surgical Revascularisation (MAIN-COMPARE) (18) showed similar findings. Data from the ASAN Medical CentreLeft MAIN Revascularisation (ASAN-MAIN) registry (19) demonstrated that stenting showed similar long-term mortality rates, $Q$ wave MI or stroke. In the Premier of Randomized Comparison of Bypass Surgery versus Angioplasty Using Sirolimus-Eluting Stent in Patients with Left Main Coronary Artery Disease (PRECOMBAT) study (20), $\mathrm{PCI}$ with sirolimus-eluting stents was noninferior to $\mathrm{CABG}$ with respect to the primary composite end point of MACCE at one year.

\section{RISK STRATIFICATION}

The SYNTAX trial (17) used a novel method to calculate angiographic complexity, known as the SYNTAX score, which incorporates the number, location and length of lesions(s), the presence of chronic total occlusions, bifurcations or trifurcations, aorto-ostial stenoses, vessel tortuosity, calcification, thrombus and diffuse disease. A higher SYNTAX score reflects greater anatomical complexity. In the LMCA subgroup of the SYNTAX trial, the patients with a low SYNTAX score had a higher rate of nondistal LMCA lesions with mainly isolated LMCA disease or LMCA disease associated with a single vessel, for which PCI could be favoured over CABG. In contrast, patients with a high SYNTAX score had a higher rate of distal LMCA lesions, the majority of which were associated with two- or three-vessel disease for which CABG may be a better alternative than PCI. Another score, the New Risk Stratification (NERS) score, showed a higher sensitivity and specificity to predict clinical outcome (21). The current ACCF/AHA/SCAI guidelines (5) provide a class IIa recommendation for PCI of left main ostial or midshaft disease when it exists in isolation or in combination with onevessel disease; a class IIb recommendation for left main distal bifurcation disease when it exists in isolation or in combination with one-vessel disease; a class IIb recommendation for any left main disease with concomitant two- or three-vessel disease and a SYNTAX score $\leq 32$; and, finally, a class III recommendation for left main disease with concomitant two- or three-vessel disease and a SYNTAX score $\geq 33$. CABG is the favoured approach for all of these scenarios (class I).

\section{ASSESSING THE SEVERITY OF LMCA OBSTRUCTION} Angiographic determination of the severity of LMCA disease is less reliable compared with other branches due to the short length of the LMCA and the diffuse nature of the disease. In case of indeterminate severity or discordance between angiographic views, further invasive evaluation, such as intravascular ultrasound (IVUS), is strongly recommended. IVUS guidance is helpful in assessing vessel size, adequate stent expansion and the absence of stent malapposition (3). A minimal luminal area $<6 \mathrm{~mm}^{2}$ has been proposed as the criterion for a significant left main obstruction according to Murray's law, based on an minimal luminal area threshold of $<4 \mathrm{~mm}^{2}$ for the LAD and LCX arteries. This cut-off has been shown to have a high sensitivity and specificity to predict a fractional flow reserve (FFR) $<0.75$ (22). A subgroup analysis from the MAIN-COMPARE registry reported that IVUS guidance was associated with improved three-year mortality compared with a conventional angiography-guided procedure (23). The current ACCF/AHA/ SCAI guidelines for PCI state that IVUS is reasonable for the assessment of angiographically indeterminate LMCA disease (class IIa) (5).

FFR, defined as the ratio of maximal blood flow in a stenotic artery to normal maximal flow, is an index of physiological significance of coronary stenosis. It can be measured by calculating the ratio of distal coronary artery pressure to aortic pressure after adenosine infusion. The FFR in a normal coronary artery is 1 . An FFR $<0.80$ has also been validated for the diagnosis of LMCA obstruction, and an FFR-guided strategy between medical therapy or CABG provides favourable longterm outcomes (24). FFR-guided PCI is associated with reduced MACE in patients with multivessel coronary artery disease at one year (25). Unlike FFR, IVUS provides morphological plaque and anatomical data that can help guide and optimize PCI. Optical coherence tomography imaging can also provide detailed plaque morphology and anatomy; however, a drawback of this technology is that ostial LMCA lesions cannot be assessed.

\section{TWO-STENT VERSUS CONVENTIONAL SINGLE-STENT STRATEGY}

In deciding on a strategy, the size of the side branch, which is always the LCX, and anatomy of the ostium are two important features. Patients with isolated LMCA disease, ostial and/or midshaft LMCA disease, LMCA disease plus single-vessel disease and SYNTAX score $<33$ have clinical outcomes with PCI that are as favourable as that for CABG. In such cases, current data support provisional single stenting as the primary strategy, although there are several scenarios in which a dedicated two-stent approach for LMCA PCI is reasonable and may be preferred. Some examples of anatomy favouring a two-stent approach include LCX disease extending $>5 \mathrm{~mm}$ from the carina, threatened closure of the LCX or when re-access to the LCX would be particularly challenging (26). Such two-stent techniques include crush, culotte, T-stenting, $\mathrm{V}$ and simultaneous kissing stents. When two stents are used, final simultaneous kissing balloon inflation at medium pressure with noncompliant balloons is crucial to optimize outcomes. However, in a meta-analysis to assess outcomes for a single stent versus a twostent strategy in the treatment of distal unprotected LMCA lesions in the DES era, a decreased risk of MACE with single-stent $(20.4 \%)$ versus two-stent strategy (32.8\%) (OR 0.51 [95\% CI 0.35 to 0.73$]$ ) (27) was reported. There was also decreased target vessel/target lesion revascularization with single-stent strategy $(10.1 \%)$ versus two-stent strategy (24.3\%) (OR 0.35 [95\% CI 0.25 to 0.49]).

\section{ANGIOGRAPHIC FOLLOW-UP}

Some recommend routine angiographic follow-up at six-month and one-year intervals; however, the SYNTAX trial demonstrated the excellent safety profile of LMCA PCI with DES in the absence of such routine angiography (17). The Unprotected Left Main Trunk Intervention Multicenter Assessment (ULTIMA) (28) registry recommended routine angiographic follow-up because there appeared to be an early hazard for mortality after BMS implantation in the first six months after discharge. A previous class IIa recommendation for angiographic follow-up was removed from the 2011 ACCF/AHA/ SCAI PCI guidelines (5).

\section{DISCUSSION}

We presented a patient with ACS who developed cardiogenic shock and significant LMCA stenosis that was managed successfully with $\mathrm{PCI}$ and DES. Cardiogenic shock in ACS is the most important cause of mortality, and early reperfusion with PCI or CABG significantly improves survival compared with initial medical stabilization (29). Although PCI has been proven to be a safe and effective treatment for most significant coronary stenoses, there are few data to support PCI in unprotected LMCA disease.

Although CABG has been considered the treatment of choice for unprotected LMCA disease revascularization, more recently, PCI has emerged as a possible substitute mode of revascularization. With the introduction of coronary stents, the outcomes of PCI in LMCA stenosis has became more favourable. The current ACCF/AHA/SCAI guidelines (5) also recommend PCI of the LMCA as a reasonable alternative to $\mathrm{CABG}$ in patients who have anatomical conditions associated with good procedural and longer-term outcomes and who are at increased risk for surgery. Moreover, studies have reported that 
PCI for unprotected LMCA disease is technically feasible in most patients and has the advantage of providing more rapid reperfusion compared with CABG, with acceptable MACE rates. In the diabetic population, the incidence of the composite of death, MI or stroke was shown to be significantly higher in PCI with DES compared with the CABG group in LMCA disease. Therefore, it has been recommended that CABG should be preferred over PCI in diabetic patients, particularly those with insulin-dependent diabetes $(3,30)$.

\section{CONCLUSION}

Patients with unprotected LMCA disease are a high-risk subgroup with a substantial mortality rate. CABG has been described as the standard of care for unprotected LMCA disease according to current guidelines. However, CABG can be time consuming and carries the risk of extensive and irreversible myocardial damage if not performed

\section{REFERENCES}

1. Goldberg S, Grossman W, Markis JE, et al. Total occlusion of the left main coronary artery: A clinical hemodynamic and angiographic profile. Am J Med 1978;64:3-8.

2. Spiecker M, Erbel R, Rupprecht HJ, Meyer J. Emergency angioplasty of totally occluded left main coronary artery in acute myocardial infarction and unstable angina pectoris - institutional experience and literature review. Eur Heart J 1994;15:602-7.

3. Fajadet J, Chieffo A. Current management of left main coronary artery disease. Eur Heart J 2012;33:36-50.

4. Kalbfleisch H, Hort W. Quantitative study on the size of coronary artery supplying areas postmortem. Am Heart J 1977;94:183-8.

5. Levine GN, Bates ER, Blankenship JC, et al. American College of Cardiology Foundation; American Heart Association Task Force on Practice Guidelines; Society for Cardiovascular Angiography and Interventions. $2011 \mathrm{ACCF} / \mathrm{AHA} / \mathrm{SCAI}$ guideline for percutaneous coronary intervention. A report of the American College of Cardiology Foundation/American Heart Association Task Force on Practice Guidelines and the Society for Cardiovascular Angiography and Interventions. J Am Coll Cardiol 2011;58:e44-122.

6. Dash D. Stenting of left main coronary artery stenosis: A to Z. Heart Asia 2013;5:18-27.

7. Park SJ, Kim YH, Lee BK, et al. Sirolimus-eluting stent implantation for unprotected left main coronary artery stenosis: Comparison with bare metal stent implantation. J Am Coll Cardiol 2005;45:351-6.

8. Valgimigli M, van Mieghem CM, Ong AT, et al. Short- and longterm clinical outcome after drug-eluting stent implantation for the percutaneous treatment of left main coronary artery disease: Insights from the Rapamycin-Eluting and Taxus Stent Evaluated at Rotterdam Cardiology Hospital registries (RESEARCH and T-SEARCH). Circulation 2005;111:1383-9.

9. Pavei A, Oreglia J, Martin G, et al. Long-term follow-up of percutaneous coronary intervention of unprotected left main lesions with drug eluting stents. Predictors of clinical outcome. EuroIntervention 2009;4:457-63.

10. Carrie D, Eltchaninoff H, Lefevre T, et al. Twelve month clinical and angiographic outcome after stenting of unprotected left main coronary artery stenosis with paclitaxel-eluting stents - results of the multicentre FRIEND registry. EuroIntervention 2009;4:449-56.

11. Dores H, Raposo L, Almeida MS, et al. Percutaneous coronary intervention of unprotected left main disease: Five-year outcome of a single-center registry. Rev Port Cardiol 2013;32:997-1004.

12. Pandya SB, Kim YH, Meyers SN, et al. Drug-eluting versus baremetal stents in unprotected left main coronary artery stenosis a meta-analysis. J Am Coll Cardiol Intv 2010;3:602-11.

13. Shemin RJ. Coronary artery bypass grafting versus stenting for unprotected left main coronary artery disease: Where lies the body of proof? Circulation 2008;118: 2326-9.

14. Garg P, Mauri I. The conundrum of late and very late stent thrombosis following drug-eluting stent implantation. Curr Opin Cardiol 2007;22:565-71.

15. Taggart DP. Coronary artery bypass graft vs. percutaneous coronary angioplasty; $\mathrm{CABG}$ on the rebound? Curr Opin Cardiol 2007;22:517-23.

16. Buszman PE, Buszman PP, Kiesz RS, et al. Early and long-term results of unprotected left main coronary artery stenting: The LE expeditiously; however, PCI allows rapid restoration of flow and can be performed more quickly than CABG and, thus, plays a vital role in preserving myocardial viability. In a developing country, such as Nepal, where cardiac surgery departments in most cases are far away and are not able to provide sufficient capacity for surgical backup, PCI remains an inevitable 'bailout' life-saving procedure. Moreover, evidence from several trails and reports suggest that PCI may provide at least equivalent results to $\mathrm{CABG}$ in the setting of less complex coronary anatomy of LMCA disease. Therefore, PCI could be a reasonable alternative to CABG in patients with the unprotected LMCA disease who have anatomical conditions associated with good procedural and longer-term outcomes and who are at increased risk for CABG surgery.

DISCLOSURES: None to report.

MANS (Left Main Coronary Artery Stenting) registry. J Am Coll Cardiol 2009;54:1500-11.

17. Morice MC, Serruys PW, Kappetein AP, et al. Outcomes in patients with de novo left main disease treated with either percutaneous coronary intervention using paclitaxel-eluting stents or coronary artery bypass graft treatment in the Synergy between Percutaneous Coronary Intervention with TAXUS and Cardiac Surgery (SYNTAX) trial. Circulation 2010;121:2645-53.

18. Seung KB, Park DW, Kim YH, et al. Stents versus coronary artery bypass grafting for left main coronary artery disease. $\mathrm{N}$ Engl J Med 2008;358:1781-92.

19. Park DW, Kim YH, Yun SC, et al. Long-term outcomes after stenting versus coronary artery bypass grafting for unprotected left main coronary artery disease: 10 -year results of bare-metal disease and 5-year results of drug-eluting stents from the ASAN-MAIN (ASAN Medical Center-Left Main Revascularization) Registry. J Am Coll Cardiol 2010;56:1366-75.

20. Park SJ, Kim YH, Park DW, et al. Randomized trial of stents versus bypass surgery for left main coronary artery disease. $\mathrm{N}$ Engl J Med 2011;364:1718-27.

21. Chen SL, Chen JP, Mintz G, et al. Comparison between the NERS (New Risk Stratification) and the SYNTAX (Synergy between Percutaneous Intervention with Taxus and Cardiac Surgery) score in outcome prediction for uncomplicated left main stenting. JACC Cardiovasc Interv 2010;3:632-41.

22. Jasti V, Ivan E, Yalamanchili V, Wongpraparut N, Leesar MA. Correlations between fractional flow reserve and intravascular ultrasound in patients with an ambiguous left main coronary artery stenosis. Circulation 2004;110:2831-6.

23. Park SJ, Kim YH, Park DW, et al. Impact of intravascular ultrasound guidance on long-term mortality in stenting for unprotected left main coronary artery stenosis. Circ Cardiovasc Interv 2009;2:167-77.

24. Hamilos M, Muller O, Cuisset T, et al. Long-term clinical outcome after fractional flow reserve-guided treatment in patients with angiographically equivocal left main coronary artery stenosis. Circulation 2009;120:1505-12.

25. Tonino PA, De Bruyne B, Pijls NH, et al. Fractional flow reserve versus angiography for guiding percutaneous coronary intervention. N Engl J Med 2009;360:213-24.

26. Hildick-Smith D, Lassen JF, Albiero R, et al. Consensus from the 5th European Bifurcation Club meeting. EuroIntervention 2010;6:34-8.

27. Karrowni W, Makki N, Dhaliwal AS, et al. Single versus double stenting for unprotected left main coronary artery bifurcation lesions: A systematic review and meta-analysis. J Inv Cardiol 2014;26:229-33.

28. Tan WA, Tamai H, Park SJ, et al. Long-term clinical outcomes after unprotected left main trunk percutaneous revascularization in 279 patients. Circulation 2001;104:1609-14.

29. Dubey L, Sharma S, Gautam M, et al. Cardiogenic shock complicating acute myocardial infarction - a review. Acta Cardiol 2011;66:691-9.

30. Luo Y, Yu X, Chen F, et al. Impact of diabetes mellitus on patients with unprotected left main coronary artery lesion disease treated with either percutaneous coronary intervention or coronary artery bypass grafting. Coron Artery Dis 2012;23:322-9. 